\title{
Alterations of thoraco-abdominal volumes and asynchronies in patients with spinal muscle atrophy type III
}

\author{
Antonella LoMauro a, ${ }^{2}, 1$, Marianna Romei ${ }^{b, 1}$, Rita Priori a , Marianna Laviola a \\ Maria Grazia D’Angelo ${ }^{b}$, Andrea Aliverti ${ }^{a}$ \\ a TBM Lab, Dipartimento di Elettronica, Informazione e Bioingegneria, Politecnico di Milano, Milano, Italy \\ ${ }^{\mathrm{b}}$ IRCCS E. Medea, Bosisio Parini, Lc, Italy
}

Accepted 4 March 2014

Available online 12 March 2014

\section{Introduction}

Spinal muscular atrophy (SMA) is an autosomal recessive disease due to a homozygous SMN1 deletion in exon 7 in most patients (Kaufmann et al., 2011; Munsat and Davies, 1992; Wee et al., 2010). SMA is characterized by degeneration of motor neurons of spinal cord that results in muscle wasting and weakness (Dubowitz, 1995; Mercuri et al., 2012).

The conventional classification of SMA into five phenotypes, namely types 0 (very severe), I (severe), II (intermediate), III (mild) and IV (adult), is based on the highest motor function achieved and the age of onset of the disease. Although this classification is clinically useful, it is not always adequate for prognosis or patients' stratification in clinical trials. In particular, the onset of lower limb muscular weakness in type III patients can be quite variable and therefore a sub-classification into type IIIA (onset of weakness before 3 years) and type IIIB (onset of weakness after 3 years) was recently proposed (Mercuri et al., 2012).
It is also well known that in SMA patients survival depends primarily on respiratory function and not necessarily on motor ability (Dubowitz, 1995; Gozal, 2000; Mellies et al., 2004; Perez et al., 1996; Russman et al., 1992; Talbot, 1999; Wang et al., 2007). Regarding respiratory muscles, there is a general consensus that the intercostal muscles are weakened and the diaphragm is relatively preserved, particularly in SMA I and SMA II. This alteration has been associated to changes in thoracic shape, leading to bell-shaped chest and pectus excavatum, and impaired ability to cough effectively (Kuzuhara and Chou, 1981; Perez et al., 1996; Schroth, 2009). In addition, abnormal thoraco-abdominal patterns of breathing characterized by asynchronous displacement of rib cage and abdomen as well as diminished contribution of either compartment to tidal volume have been reported only in SMA II patients and only in supine position by respiratory inductive plethysmography (Perez et al., 1996) and early versions of optoelectronic plethysmography (Lissoni et al., 1996).

Very little attention has been paid to possible alterations in the respiratory pattern in type III patients (Bach et al., 2012; Piepers et al., 2008). For this reason, we aimed to study SMA type III patients in terms of chest wall and respiratory muscle function. More specifically, our purpose was to investigate if SMA IIIA and SMA IIIB have or do not have a different respiratory muscle action resulting in altered chest wall volumes and asynchronies and therefore

\footnotetext{
* Corresponding author. Tel.: +39 223999025; fax: +39 223990000.

E-mail address: antonella.lomauro@polimi.it (A. LoMauro).

1 The two authors equally contributed to the study.
} 
if the proposed sub-classification is feasible also for respiratory function.

\section{Materials and methods}

\subsection{Subjects}

Eighteen patients with a defined diagnosis of SMA (clinical and neurophysiological signs, molecular alterations in the Survival Motor Neuron 1, SMN1 gene) (Munsat and Davies, 1992; Wee et al., 2010) and 18 healthy age-matched volunteers were included in the study.

Demographic and anthropometric data of both groups are reported in Table 1.

SMA patients' population included 4 patients with SMA type II. They never acquired ambulation; three of them underwent spinal fusion and one had severe scoliosis (Cobb angle $>40^{\circ}$ ); all of them were treated with cough assisted devices and one was under nocturnal non-invasive mechanical ventilation.

Among the 14 patients with SMA type III, 5 were classified as SMA IIIA and 9 as SMA IIIB. In the SMA IIIA group, all patients lost ambulation in the first 3-4 years of life, two underwent spinal fusion and all the others were presenting severe scoliosis. In the SMA IIIB group, only two patients lost ambulation at the age of 17 and 21 years, respectively.

All patients were evaluated with the 20 items Hammersmith functional motor scale (HFMS) for children with SMA (Main et al., 2003; Mercuri et al., 2006) and the scores for SMA II, IIIA and IIIB patients are reported in Table 1.

None of the SMA patients was under salbutamol treatment. All subjects or parents signed a written informed consent form, as approved by the Local Ethical Committee of IRCCS "E. Medea" Institute according to the declaration of Helsinki.

\subsection{Measurements, protocol and data analysis}

\subsubsection{Pulmonary function tests}

Pulmonary function analysis was performed in all cooperative patients, i.e. in 15 out of 18 . Measurements of forced vital capacity (FVC), forced expiratory volume in one second $\left(\mathrm{FEV}_{1}\right)$ and Peak Expiratory Flow (PEF) were performed in seated position with a flowmeter attached to a flanged rubber mouthpiece with the nose occluded (Vmax series 22, SensorMedics, Yorba Linda, USA). Subdivisions of lung volume (Functional Residual Capacity, FRC; Residual Volume, RV and Total Lung Capacity, TLC) were measured by the nitrogen washout technique (Vmax series 22, SensorMedics, Yorba Linda, CA).

\subsubsection{Optoelectronic Plethysmography measurements}

Total and compartmental chest wall volumes were measured by Opto-Electronic Plethysmography (OEP System; BTS, Milan, Italy) in all subjects (Aliverti et al., 2000, 2001; Cala et al., 1996). The sys-tem, based on eight infrared video cameras working at a sampling rate of $60 \mathrm{~Hz}$, computes the 3D coordinates of retroreflective mark-ers placed, according to specific anatomical points, over the chest wall surface of the subject. To study supine position, 52 markers were used in both healthy controls and patients. To study seated position, either 52 or 89 markers have been used, depending on the ability of the subject to maintain an erect position of the trunk while sitting without a back support.

The 3D coordinates of the markers were used to compute the volume of total chest wall $\left(\Delta V_{\mathrm{CW}}\right)$ and its three thoraco-abdominal compartments, namely pulmonary rib cage $\left(\Delta V_{\mathrm{RC}, \mathrm{P}}\right)$, abdominal rib cage $\left(\Delta V_{\mathrm{RC}, \mathrm{A}}\right)$ and abdomen $\left(\Delta V_{\mathrm{AB}}\right)$, during quiet breathing, inspiratory capacity and maximal voluntary cough in seated and supine positions (Romei et al., 2010).

2.2.2.1. Quiet breathing. After a period of adaptation to the experimental conditions, spontaneous quiet breathing (QB) was measured during three minutes. During QB, the following parameters were calculated from chest wall volume measurements over a period of about one minute taken in the middle of the recorded signals: minute ventilation $\left(V_{\mathrm{E}}\right)$, respiratory rate $(\mathrm{RR})$, tidal volume $\left(V_{\mathrm{T}}\right)$ and percentage contribution to $V_{\mathrm{T}}$ of pulmonary rib cage, abdominal rib cage and abdomen (\% $\Delta V_{\mathrm{RC}, \mathrm{P}} \% \Delta V_{\mathrm{RC}, \mathrm{A}}$ and $\% \Delta V_{\mathrm{AB}}$, respectively). In addition, $V_{\mathrm{E}}$ and $V_{\mathrm{T}}$ were normalized by body weight in order to take into account possible body size differences.

2.2.2.2. Inspiratory capacity. After QB measurements, patients and healthy controls were asked to perform a full inspiration to total lung capacity starting from end-expiratory volume (inspiratory capacity, IC). The maneuver was repeated twice with an interval of at least $30 \mathrm{~s}$. On the best maneuver, i.e. the maneuver providing the greatest IC value, total chest wall volume variations and percentage contribution of the three compartments were calculated (Romei et al., 2012).

2.2.2.3. Cough. Finally, patients and healthy controls were asked to perform three single maximal voluntary coughs. On the best maneuver, i.e. the maneuver providing the highest peak value of the signal obtained as the time derivative of chest wall volume, the points of start of inspiration and end of inspiration were identi-fied on chest wall tracings in order to calculate inspiratory cough phase (ICP) as the difference between these two points. In the same instants, the percentage contribution of the different compartments to total chest wall volume variations was computed (LoMauro et al., 2013).

2.2.2.4. Rib cage and thoraco-abdominal asynchronies. During $\mathrm{QB}$, the phase shift $(\theta)$ between volume variations of two compartments was calculated with Lissajous loop analysis, as previously described in detail (Priori et al., 2013). In brief, a phase shift of $0^{\circ}$ means that the volume variations of the considered compartments are completely synchronous, while $180^{\circ}$ describes a completely paradoxical motion. Asynchronies within the rib cage $\left(\theta_{\mathrm{RC}}\right)$ and between rib cage and abdomen ('thoraco-abdominal', $\theta_{\mathrm{TA}}$ ) were computed as phase shift between $\Delta V_{\mathrm{RC}, \mathrm{P}}$ and $\Delta V_{\mathrm{RC}, \mathrm{A}}$ and between $\Delta V_{\mathrm{RC}, \mathrm{P}}$ and

$\Delta V_{\mathrm{AB}}$, respectively. By convention, a positive angle means that pulmonary rib cage expansion is leading on the expansion of the other compartment (either abdominal rib cage or abdomen).

\subsection{Statistical analysis}

Anthropometric, clinical, spirometric, lung function, ventilatory and asynchrony parameters of the four groups were compared using one-way Analysis of Variance (ANOVA) with disease as independent factor. Total and compartmental chest wall volume variations during $\mathrm{QB}, \mathrm{ICP}$ and IC were compared using one-way Analysis of Variance (ANOVA) with maneuver as independent factor. When normality test failed, Kruskal-Wallis one-way ANOVA on Ranks was used. Post-hoc tests were based on Holm-Sidak and Dunn's methods, respectively, for parametric and non parametric ANOVA. Linear correlation analysis was performed between individual HFMS score data and FVC, abdominal percentage contribution to tidal volume during quiet breathing, to inspired volume preceding cough and to inspiratory capacity in supine position. Data in tables are expressed as median and range, and in figures as median and either individual data or 5-95th percentiles. Significance was determined by $p<0.05$. 
Table 1

Anthropometric, functional, spirometric and lung volume data.

\begin{tabular}{|c|c|c|c|c|c|c|c|c|}
\hline & \multicolumn{2}{|c|}{ SMA II } & \multicolumn{2}{|c|}{ SMA IIIA } & \multicolumn{2}{|c|}{ SMA IIIB } & \multicolumn{2}{|c|}{ Control } \\
\hline$N$ & \multicolumn{2}{|c|}{$4(2 \mathrm{M}, 2 \mathrm{~F})$} & \multicolumn{2}{|c|}{$5(3 \mathrm{M}, 2 \mathrm{~F})$} & \multicolumn{2}{|c|}{$9(7 \mathrm{M}, 2 \mathrm{~F})$} & \multicolumn{2}{|c|}{$18(15 \mathrm{M}, 3 \mathrm{~F})$} \\
\hline Age [years] & 22 & $(12-40)$ & 14 & $(5-42)$ & 21 & $(6-44)$ & 25 & $(7-40)$ \\
\hline Weight [kg] & 45 & $(44-73)$ & 62 & $(14-73)$ & 58 & $(18-85)$ & 61 & $(24-84)$ \\
\hline Height $[\mathrm{cm}]$ & 152 & $(150-155)$ & 150 & $(108-167)$ & 167 & $(115-175)$ & 173 & $(126-181)$ \\
\hline HFMS score ${ }^{\mathrm{a}}$ & $8^{*}$ & $(2-13)$ & 16 & $(8-39)$ & 39 & $(28-40)$ & - & - \\
\hline \multicolumn{9}{|l|}{ Spirometric data } \\
\hline (available data) & 3 & & 3 & & 9 & & & \\
\hline FVC [L] & 2.4 & $(1.8-2.7)$ & 3.24 & $(0.99-3.54)$ & 3.56 & $(2.74-4.26)$ & & \\
\hline FVC [\%pred] & 76 & $(56-89)$ & 77 & $(55-85)$ & 84 & $(70-113)$ & & \\
\hline $\mathrm{FEV}_{1}[\mathrm{~L}]$ & 2.16 & $(1.44-2.51)$ & 2.97 & $(0.91-3.1)$ & 3.14 & $(2.46-3.86)$ & & \\
\hline $\mathrm{FEV}_{1}[\%$ pred] & 79 & $(51-99)$ & 85 & $(60-87)$ & 87.5 & $(67-106)$ & & \\
\hline $\mathrm{FEV}_{1} / \mathrm{FVC}[\%]$ & 90 & $(78-93)$ & 91 & $(88-91)$ & 83.5 & $(79-98)$ & & \\
\hline $\mathrm{PEF}\left[\mathrm{Lsec}^{-1}\right]$ & 4.85 & $(3.54-5.07)$ & 6.29 & $(2.68-6.94)$ & 5.19 & $(3.94-7.36)$ & & \\
\hline PEF [\%pred] & 75 & $(46-93)$ & 75 & $(73-79)$ & 69.5 & $(44-82)$ & & \\
\hline \multicolumn{9}{|l|}{ Lung volume data } \\
\hline (available data) & 3 & & 3 & & 9 & & & \\
\hline TLC $[\mathrm{L}]$ & 4.43 & $(2.83-4.81)$ & 5.16 & $(1.66-5.83)$ & 5.35 & $(4.44-6.01)$ & & \\
\hline TLC [\%pred] & 100 & $(58-119)$ & 87 & $(65-93)$ & 94.5 & $(81-104)$ & & \\
\hline $\mathrm{RV}[\mathrm{L}]$ & 1.86 & $(0.99-1.96)$ & 1.62 & $(0.67-2.3)$ & 1.51 & $(1.19-2.43)$ & & \\
\hline RV [\%pred] & 149 & $(61-201)$ & 102 & $(99-122)$ & 118 & $(76-172)$ & & \\
\hline FRC $[\mathrm{L}]$ & 2.2 & $(1.2-2.2)$ & 2.18 & $(0.87-2.77)$ & 2.63 & $(1.73-3.04)$ & & \\
\hline FRC [\%pred] & 88 & $(43-118)$ & 77 & $(72-87)$ & 94 & $(63-110)$ & & \\
\hline
\end{tabular}

a HFMS (Hammersmith functional motor scale) score ranges from 0 (all activities failed) to 40 (all activities achieved).

$p<0.05$ vS SMA IIIB

\section{Results}

\subsection{Anthropometric, functional, spirometric and lung volume data}

As shown in Table 1, all groups were homogeneous in age and their anthropometric characteristics were similar $(p>0.05)$. HFMS scores were significantly lower in SMA II than in SMA IIIB patients, while their range in SMA IIIA patients was very large.

The overall group of SMA patients showed a mild restrictive syndrome, with slightly reduced values of FVC, FEV 1 , PEF and FRC and corresponding slightly increased values of $\mathrm{FEV}_{1} / \mathrm{FVC}$ and RV (Table 1). No statistically significant differences were present among SMA groups. Lung function of healthy controls is not reported since it was measured only in a few, due to the unavailability of the instrumentation at the moment of OEP data acquisition.

\subsection{Ventilatory pattern}

Both in the seated and supine positions, during quiet breath-ing no significant differences in any ventilatory parameter (minute ventilation and tidal volume, both absolute and body-weight normalized, and respiratory rate) were found between SMA patients and controls (Table 2).

\subsection{Chest wall volume}

In the seated position, all type II SMA patients were analysed with 52 markers, 3 out of 5 type IIIA patients with 52 markers, while all type IIIB patients and controls with 89 markers.

Median and individual values of chest wall volume variations during quiet breathing, inspiratory cough phase and inspiratory capacity for the four considered groups and for the two considered postures are reported in Fig. 1. Although in all groups median chest wall expansion increased passing from $\mathrm{QB}$ to ICP and IC, only in SMA IIIB patients and controls median values of IC were significantly greater than ICP $(p<0.05)$, that is chest wall volume variations were similar during ICP and IC in SMA II and IIIA patients.

\subsection{Thoraco-abdominal volume contributions}

Percentage contributions of pulmonary rib cage and abdomen during quiet breathing, inspiratory cough phase and inspiratory capacity for the four considered groups and for the two considered postures are reported in Figs. 2 and 3.

In contrast to the healthy subjects, who progressively increased $\% \Delta V_{\mathrm{RC}, \mathrm{P}}$ and consequently reduced $\% \Delta V_{\mathrm{AB}}$ passing from $\mathrm{QB}$ to ICP and IC, all SMA patients showed a relatively constant thoracoabdominal contribution of the different compartments to total volume change during the different maneuvers ( $\mathrm{QB}, \mathrm{ICP}$ and IC). In SMA II and SMA IIIA patients, \% $\Delta V_{\mathrm{RC}, \mathrm{P}}$ was invariantly lower than $\% \Delta V_{\mathrm{AB}}$, while in SMA IIIB \% $\Delta V_{\mathrm{AB}}$ was lower than $\% \Delta V_{\mathrm{RC} . \mathrm{P}}$, being more similar to controls $(p<0.05)$.

Variations of $\% \Delta V_{\mathrm{RC}, \mathrm{A}}$ (not shown in Figs. 2 and 3) between $\mathrm{QB}$, ICP and IC within the different groups and between groups were similar to those of the $\% \Delta V_{\mathrm{RC}, \mathrm{P}}$ in both postures.

\subsection{Chest wall asynchronies}

Rib cage asynchrony, quantified as the phase shift $\left(\theta_{\mathrm{RC}}\right)$ between $\Delta V_{\mathrm{RC}, \mathrm{p}}$ and $\Delta V_{\mathrm{RC}, \mathrm{A}}$, and thoraco-abdominal asynchrony, quantified as the phase shift $\left(\theta_{\mathrm{TA}}\right)$ between $\Delta V_{\mathrm{RC}, \mathrm{P}}$ and $\Delta V_{\mathrm{AB}}$, during quiet breathing in the four considered groups are reported for both considered postures in Fig. 4.

In the supine position, SMA II and SMA IIIA patients showed significantly more negative values of $\theta_{\mathrm{RC}}$ and $\theta_{\mathrm{TA}}$ than SMA IIIB and controls. Again, no differences were found between SMA II and SMA IIIA patients and between SMA IIIB and controls. In seated position, although a similar trend was observed, no statistically significant differences were found probably due to the high intersubject variability of $\theta_{\mathrm{RC}}$ and $\theta_{\mathrm{TA}}$.

Fig. 5 illustrates representative examples of thoraco-abdominal volume variations occurring in supine position during quiet breathing in four subjects belonging to the considered groups. With decreasing severity of the disease, the volume change of the pulmonary rib cage from the beginning to the end of inspiration (gray areas in figure) progressively increases, while the volume change of the abdomen progressively decreases. In SMA II and SMA IIIA, 
Table 2

Ventilatory pattern during quite breathing in seated and supine position.

\begin{tabular}{|c|c|c|c|c|c|c|c|c|c|}
\hline \multirow[b]{2}{*}{ Tidal volume } & \multirow[b]{2}{*}{ Seated } & \multicolumn{2}{|l|}{ SMA II } & \multicolumn{2}{|c|}{ SMA IIIA } & \multicolumn{2}{|l|}{ SMA IIIB } & \multicolumn{2}{|l|}{ Control } \\
\hline & & 0.35 & $(0.21-0.53)$ & 0.27 & $(0.22-0.43)$ & 0.46 & $(0.2-1.78)$ & 0.46 & $(0.25-0.93)$ \\
\hline [L] & Supine & 0.39 & $(0.26-0.53)$ & 0.24 & $(0.22-0.33)$ & 0.36 & $(0.17-0.5)$ & 0.33 & $(0.18-0.8)$ \\
\hline Tidal volume/weight & Seated & 6.97 & $(5.5-12.1)$ & 7 & $(3.3-19)$ & 7.5 & $(3.7-32.3)$ & 5.6 & $(2.4-21.3)$ \\
\hline$\left[\mathrm{mL} \mathrm{kg}^{-1}\right]$ & Supine & 7.62 & $(2.88-12)$ & 4.6 & $(3.2-17.3)$ & 6.1 & $(3.9-10.5)$ & 8.9 & $(5.4-23.4)$ \\
\hline \multirow{2}{*}{ Minute Ventilation [L] } & Seated & 6 & $(4.6-6.9)$ & 4.55 & $(3.1-8.3)$ & 8.2 & $(3.7-13.2)$ & 8.7 & $(5.4-15.8)$ \\
\hline & Supine & 6.4 & $(5.6-9.1)$ & 4.87 & $(3.5-6.6)$ & 5.9 & $(4.1-11.1)$ & 5.9 & $(3.9-16.6)$ \\
\hline Minute Ventilation/weight & Seated & 0.13 & $(0.06-0.16)$ & 0.13 & $(0.04-0.3)$ & 0.14 & $(0.06-0.3)$ & 0.14 & $(0.1-0.4)$ \\
\hline$\left[\mathrm{Lmin}^{-1} \mathrm{~kg}^{-1}\right]$ & Supine & 0.13 & $(0.09-0.2)$ & 0.11 & $(0.07-0.3)$ & 0.09 & $(0.07-0.25)$ & 0.1 & $(0.05-0.48)$ \\
\hline Respiratory rate & Seated & 17.8 & $(12.8-22.7)$ & 13.9 & $(13.9-20.8)$ & 17.5 & $(8.7-23.6)$ & 18.5 & $(8.3-29.7)$ \\
\hline$\left[\min ^{-1}\right]$ & Supine & 16.4 & $(14.9-25.7)$ & 18.1 & $(14.8-29.8)$ & 17.8 & $(12.1-24.7)$ & 19.6 & $(6.9-23.9)$ \\
\hline
\end{tabular}

but not in SMA IIIB, an inspiratory paradoxical indrawing of the pulmonary rib cage is evident. The bottom panels of the same figure show that slope of $\Delta V_{\mathrm{RC}, \mathrm{P}} \mathrm{VS} \Delta V_{\mathrm{AB}}$ dynamic loops changes from negative (SMA II and SMA IIIA) to positive (SMA IIIB and healthy control), while the direction of the loops is always counterclockwise.

\subsection{Correlation between motor and respiratory functional data}

Fig. 6 shows the relationships existing between individual values of HFMS score and FVC, abdominal contribution to tidal volume during $\mathrm{QB}$ and to IC in supine position. In these plots, the clusters of SMA II and SMA IIIB data points were well separated, while SMA IIIA data were spread in between. Significant linear correlations, however, were found between motor and respiratory functional data: $r^{2}=0.478, p=0.006$ (HFMS vs FVC); $r^{2}=0.296, p=$ 0.020 (HFMS vs abdominal contribution to tidal volume during $\mathrm{QB}) ; r^{2}=0.439, p=0.003$ (HFMS vs abdominal contribution to IC). The relationship between HFMS and abdominal contribution to inspired volume preceding cough was instead just below the significance level $\left(r^{2}=0.233, p=0.058\right)$ and it is not shown in the figure.

\section{Discussion}

The present study provides a detailed description of respiratory function in terms of spirometry, thoraco-abdominal volumes and asynchronies in two non-lethal forms of spinal muscular atrophy, namely SMA II and SMA III sub-classified in type IIIA and IIIB as recently proposed (Mercuri et al., 2012). The main result of this study is that SMA IIIA and SMA II patients are characterized by similar respiratory pattern, whereas SMA IIIB patients are similar to the control group, and that therefore the sub-classification of type III is valid not only for motor ability, but also for respiratory function.

In order to verify the general consensus that SMA is characterized by weakened intercostal muscles and a relatively spared diaphragm, we have measured total and compartmental chest wall volumes during spontaneous quiet breathing and during two
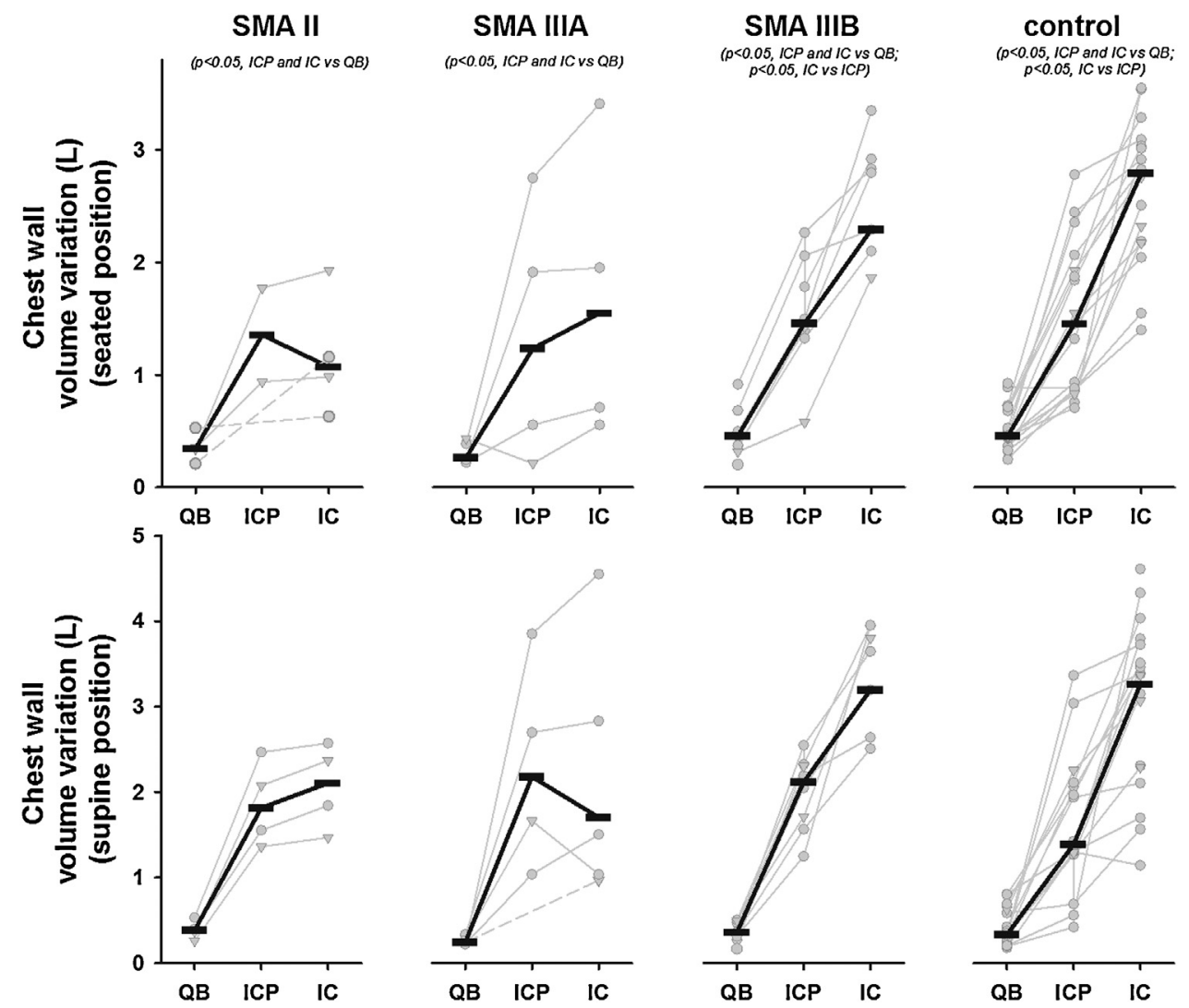

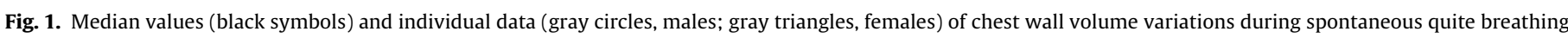

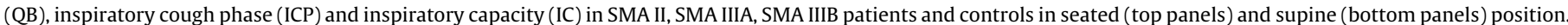



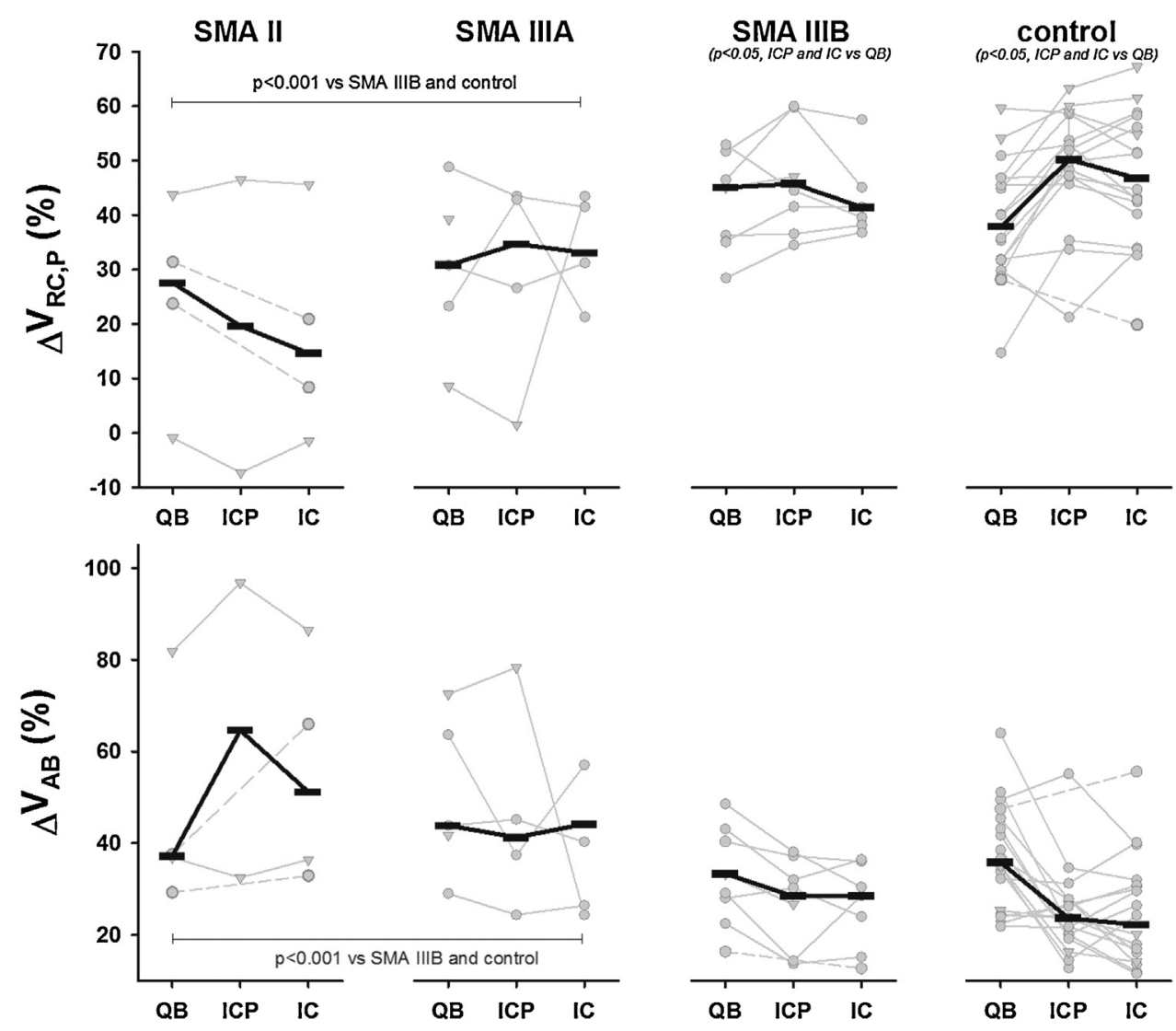

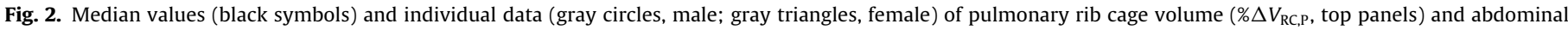

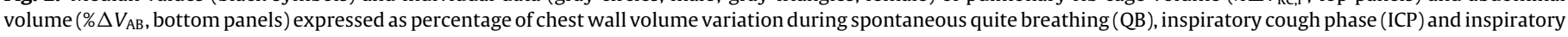
capacity (IC) in SMA II, SMA IIIA, SMA IIIB patients and controls in seated position.

voluntary respiratory maneuvers, i.e. cough and inspiratory capacity, in two different postures in patients and healthy subjects used as control.

In SMA II and SMA IIIA, in the seated position patients were not able to gradually increase inspired volume from QB to IC as SMA IIIB and controls do. In the supine position, all SMA patients and con-trols significantly increased chest wall expansion passing from QB to the respiratory maneuvers (ICP and IC). SMA II and SMA IIIA, how-ever, were characterized by reduced volume reserve performing ICP and IC (Fig. 1). These results suggest a pattern of restricted respiratory system which is supported by $\mathrm{FEV}_{1} / \mathrm{FVC}$ values significantly higher than $80 \%$.

When the contribution of thoraco-abdominal compartments are considered, an altered muscle action strikingly emerges and two different patterns are found, one belonging to type IIIA and type II patients and another to type IIIB and controls. In particular, the poor pulmonary rib cage expansion observed in SMA II and SMA IIIA patients confirms that in these patients intercostal muscles are weak and the diaphragm not only is spared, but it provides the prevalent contribution to ventilation, as suggested by the signif-icantly high abdominal contribution to tidal volume, particularly in the supine position. Interestingly, we recently found that in patients with Duchenne muscular dystrophy (DMD) with compa-rable age to our SMA groups, abdominal expansion is significantly reduced in the supine position during the three conditions here studied, namely quite breathing (Lo Mauro et al., 2010), inspiratory capacity (Romei et al., 2012) and cough (LoMauro et al., 2013). The diaphragm, therefore, seems to be preserved in SMA II and SMA III, and impaired in DMD patients. The supine posture, that is well known to have a beneficial effect on the diaphragm by stretching its fibers to an optimal length, emphasizes these differences.
During sleep, when supine position is commonly adopted, these differences in diaphragm function between SMA and DMD patients could be therefore the cause of the lower incidence of nocturnal mechanical ventilator dependence in SMA than in DMD.

Another original finding of the present study is that in SMA II and SMA IIIA patients, a higher degree of asynchrony, compared to SMA IIIB and controls, is present between the upper (pulmonary) and the lower (abdominal) rib cage expansion and between upper rib cage and abdominal expansion. These results once more suggest that in SMA II and SMA IIIA the weakened inspiratory rib cage muscle action lags behind diaphragmatic action. The marked within-rib cage asynchrony determines the energetically inefficient rib cage distortion, especially in the supine position. The very similar degrees of $\theta_{\mathrm{RC}}$ and $\theta_{\mathrm{TA}}$ asynchronies observed in SMA II and SMA IIIA groups indicate that in these patients the abdominal rib cage expands together with the abdomen, a further confirma-tion that diaphragm acts normally to displace both the lower rib cage and the abdominal compartments. Our findings are in agree-ment with those reported by Perez et al. (1996) who observed that in patients with SMA, thoraco-abdominal asynchronies are often caused by the paralysis of the rib cage muscles in presence of normal diaphragmatic activity. Because of the paralysis of the inspiratory rib cage muscles, the rib cage is neither stabilized nor expanded during inspiration, and the inspiratory negative intrathoracic pressure created by the diaphragm contraction can cause the rib cage compartment to have a decreased motion or even present an inward paradoxical motion. Interestingly, the same authors observed that, in contrast, in patients with myopathies including DMD, the diaphragm may be partially paralyzed, and therefore the inspiratory rib cage muscles assume the leading role in generating inspiratory negative pressure. In these conditions the abdominal 

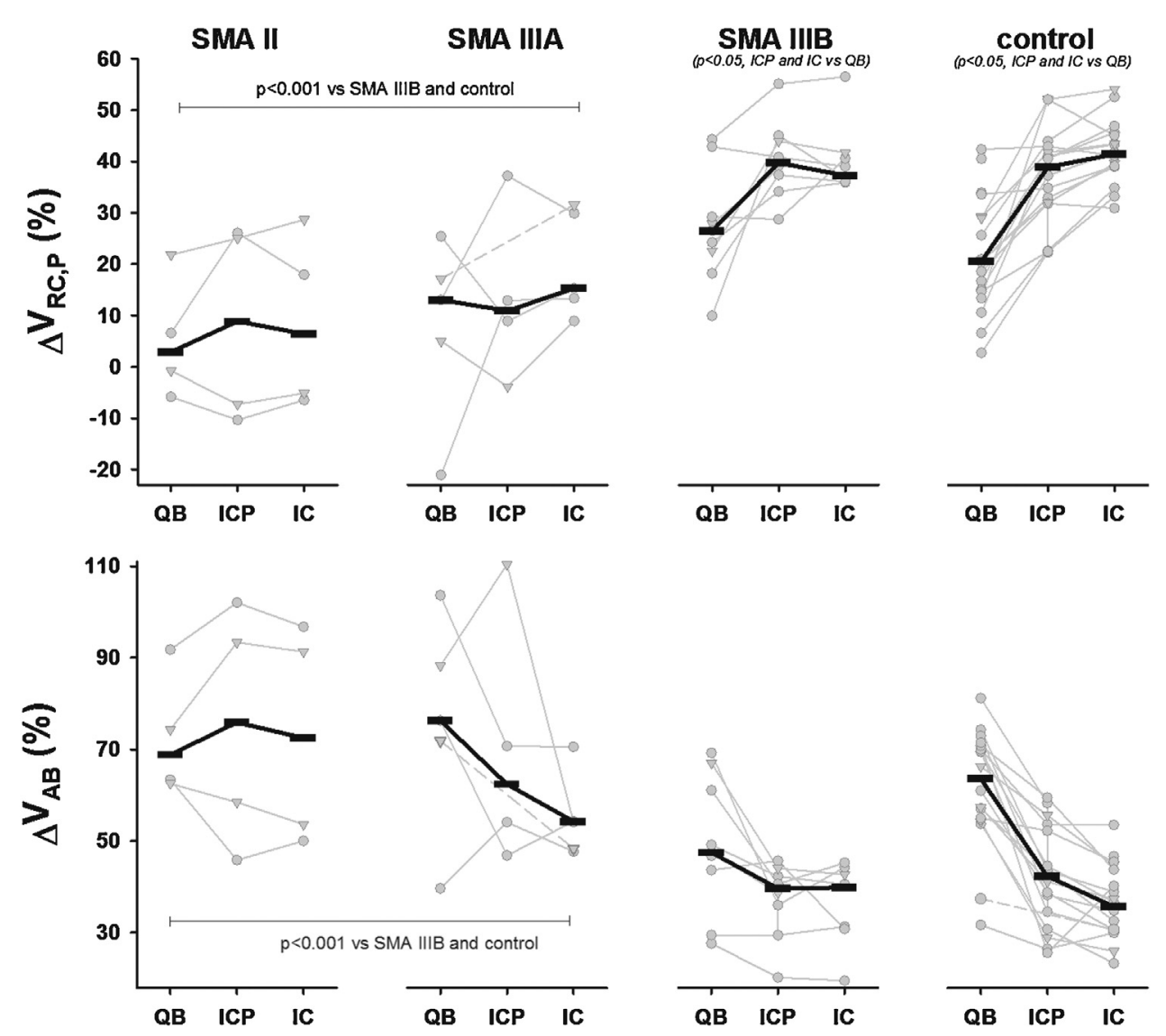

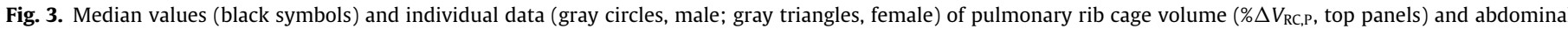

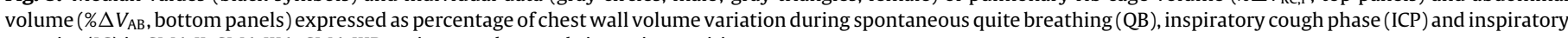
capacity (IC) in SMA II, SMA IIIA, SMA IIIB patients and controls in supine position.

compartment shows a decreased motion or, in the most advanced stages, presents an inward paradoxical motion (Lo Mauro et al., 2010; Romei et al., 2012).

The main limitation of the present work is the relatively low number of patients analyzed. In addition, to study seated posture, either 52 or 89 markers have been used and one could ques-tion whether the use of different number of markers and the corresponding geometrical model to calculate volumes contribute to the larger variability observed in type II and IIIA groups and more in general could influence the results. All type II patients were analysed with the same number of markers, and therefore the observed larger variability cannot be due to this factor in this group. In a recently published paper, Romei et al. (2010) report that using either 52 or 89 markers in healthy subjects determines a difference of about $7 \%$ in rib cage and abdominal contribution to tidal volume during quiet breathing. This value is far below the differences found between type II/type IIIA and type IIIB/controls in seated position. As the same number of markers was used in all groups, this limitation is not present in the analysis of supine posture, where different patterns were again found between type II/type IIIA and type IIIB/control groups.
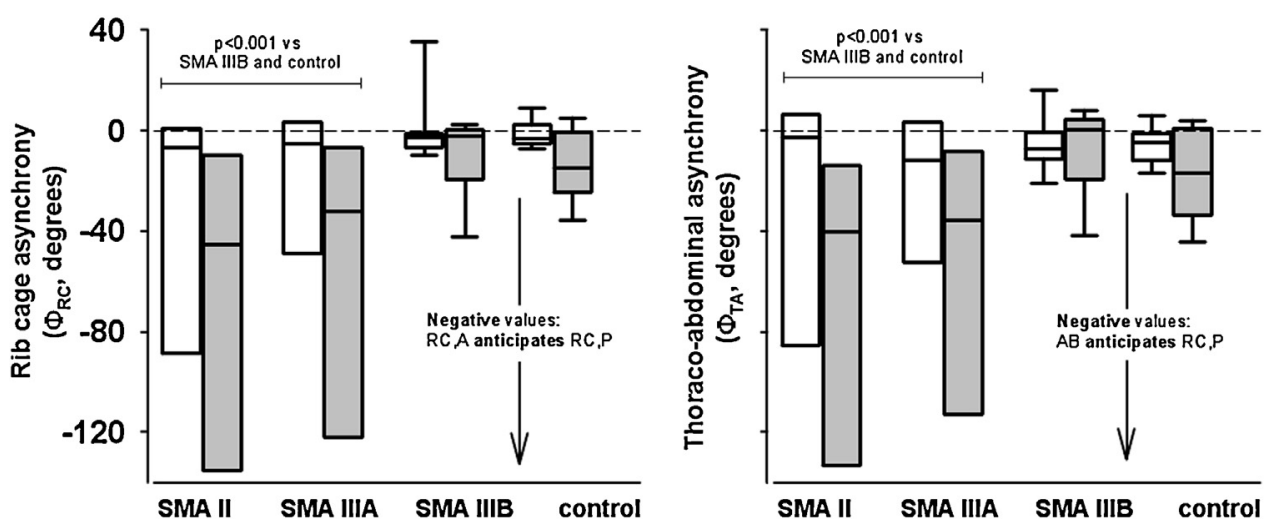

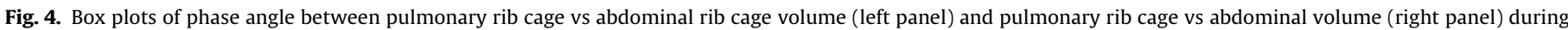

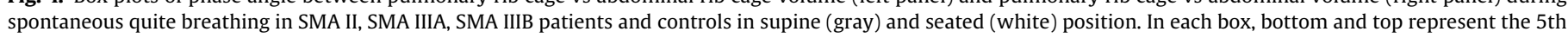
and 95th percentiles and the intermediate line represents the median. 


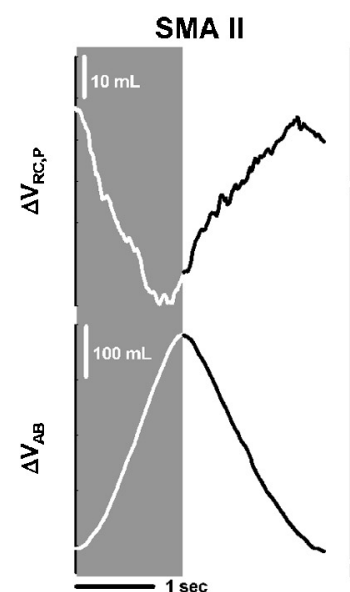

SMA IIIA
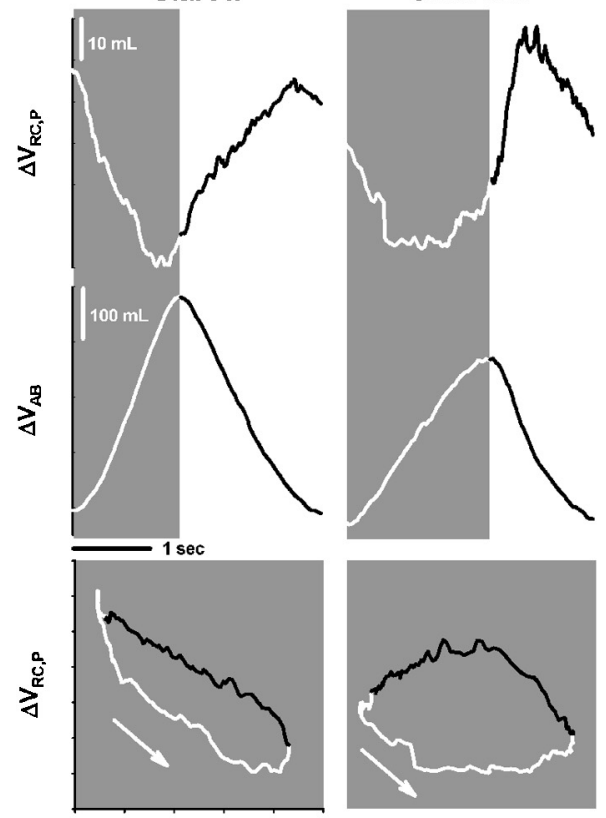

SMA IIIB
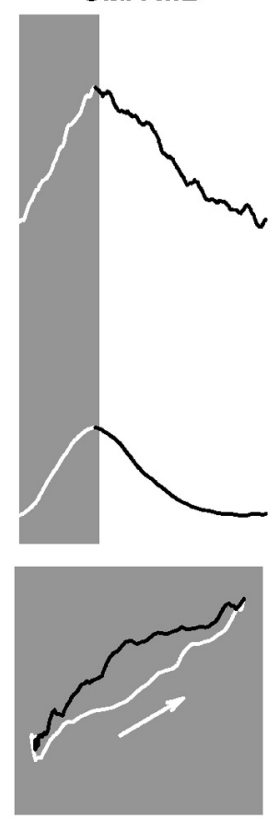

control
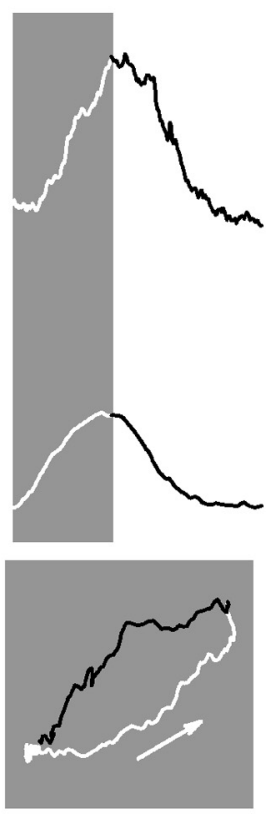

$\Delta \mathbf{V}_{A B}$

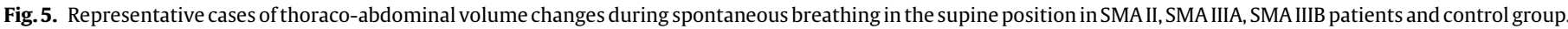

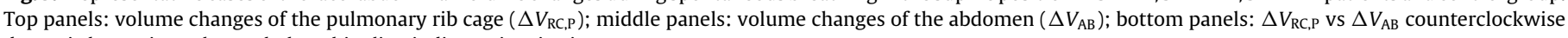
dynamic loops; in each panel, the white line indicates inspiration.
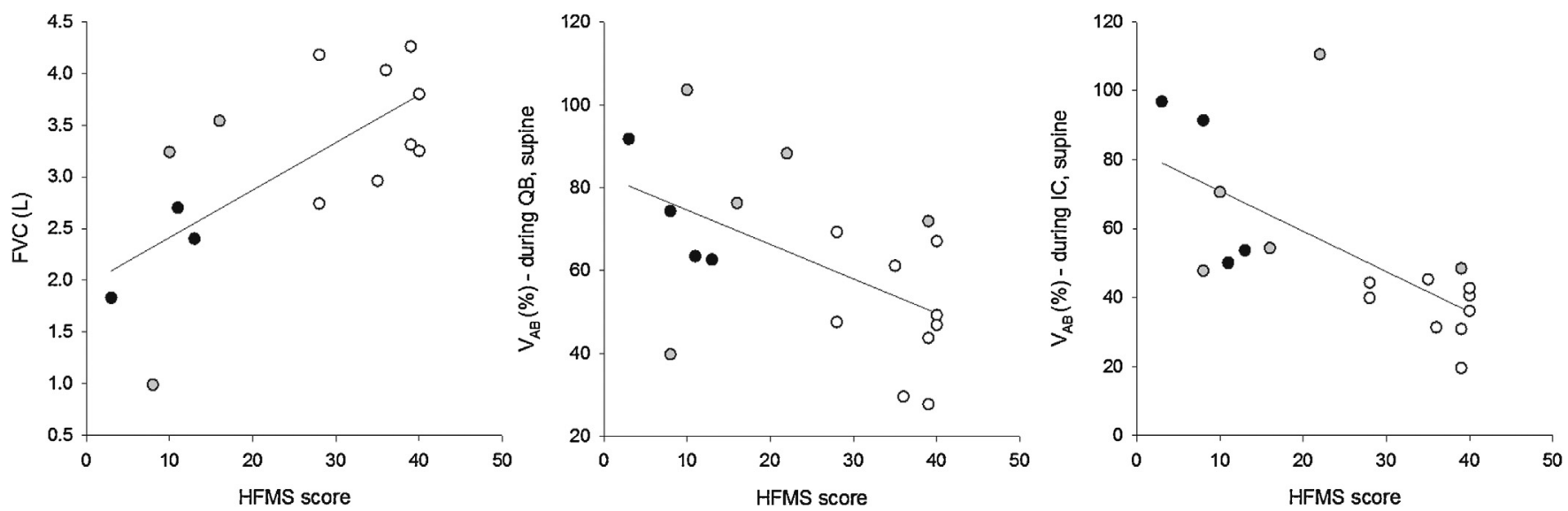

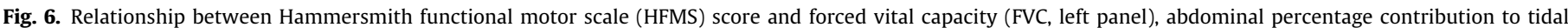

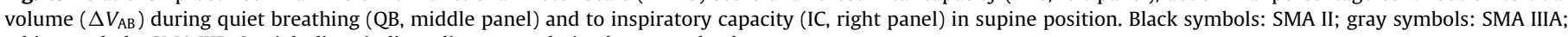
white symbols: SMA IIIB. Straight lines indicate linear correlation between the data.

The original aspect of the present study is the focus on patients with SMA type III, a form of SMA whose respiratory implications have received little attention in the past. We showed that SMA III cannot be actually considered as a homogeneous group also from the respiratory point of view. Despite the limited size of our cohort, our results also indicate that significant linear correlations exist between motor and respiratory functional data. Although these findings need to be confirmed on larger groups of patients, we believe that they should be strongly considered in designing future clinical trials.

\section{References}

Aliverti, A., Dellaca, R., Pelosi, P., Chiumello, D., Pedotti, A., Gattinoni, L., 2000. Optoelectronic plethysmography in intensive care patients. Am. J. Respir. Crit. Care Med. 161 (5), 1546-1552.
Aliverti, A., Dellaca, R., Pelosi, P., Chiumello, D., Gattinoni Pedotti, A., 2001. Compartmental analysis of breathing in the supine and prone positions by optoelectronic plethysmography. Ann. Biomed. Eng. 29 (1), 60-70.

Bach, J.R., Tuccio, M.C., Khan, U., Saporito, L.R., 2012. Vital capacity in spinal muscular atrophy. Am. J. Phys. Med. Rehabil. 91 (6), 487-493.

Cala, S.J., Kenyon, C.M., Ferrigno, G., Carnevali, P., Aliverti, A., Pedotti, A., Macklem, P.T., Rochester, D.F., 1996. Chest wall and lung volume estimation by optical reflectance motion analysis. J. Appl. Physiol. 81, 2680-2689.

Dubowitz, V., 1995. Chaos in the classification of SMA: a possible resolution. Neuromuscul. Disord. 5 (1), 3-5.

Gozal, D., 2000. Pulmonary manifestations of neuromuscular disease with special reference to Duchenne muscular dystrophy and spinal muscular atrophy. Pediatr. Pulmonol. 29 (2), 141-150.

Kaufmann, P., McDermott, M.P., Darras, B.T., Finkel, R., Kang, P., Oskoui, M., Constantinescu, A., Sproule, D.M., Foley, A.R., Yang, M., Tawil, R., Chung, W., Martens, B. Montes, J., O’Hagen, J., Dunaway, S., Flickinger, J.M., Quigley, J., Riley, S., Glanzman, A.M., Benton, M., Ryan, P.A., Irvine, C., Annis, C.L., Butler, H., Caracciolo, J., Montgomery, M., Marra, J., Koo, B., De Vivo, D.C., Muscle Study Group; Pediatric Neuromuscular Clinical Research Network for Spinal Muscular Atrophy, 2011. Observational study of spinal muscular atrophy type 2 and 3: functional outcomes over 1 year. Arch. Neurol. 68 (6), 779-786. 
Kuzuhara, S., Chou, S.M., 1981. Preservation of the phrenic motoneurons in Werdnig-Hoffmann disease. Ann. Neurol. 9 (5), 506-510.

Lissoni, A., Aliverti, A., Molteni, F., Bach, J.R., 1996. Spinal muscular atrophy: kinematic breathing analysis. Am. J. Phys. Med. Rehabil. 75 (5), 332-339.

Lo Mauro, A., D’Angelo, M.G., Romei, M., Motta, F., Colombo, D., Comi, G.P., Pedotti, A., Marchi, E., Turconi, A.C., Bresolin, N., Aliverti, A., 2010. Abdominal volume contribution to tidal volume as an early indicator of respiratory impairment in Duchenne muscular dystrophy. Eur. Respir. J. 35 (5), 1118-1125.

LoMauro, A., Romei, M., D’Angelo, G., Aliverti, A., 2013. Inefficient cough in Duchenne muscular dystrophy (DMD). Pediatr. Pulmonol. http://dx.doi.org/10.1002/ppul.22836.

Main, M., Kairon, H., Mercuri, E., Muntoni, F., 2003. The Hammersmith functional motor scale for children with spinal muscular atrophy: a scale to test ability and monitor progress in children with limited ambulation. Eur. J. Paediatr. Neurol. 7, 155-159.

Mellies, U., Dohna-Schwake, C., Stehling, F., Voit, T., 2004. Sleep disordered breathing in spinal muscular atrophy. Neuromuscul. Disord. 14 (12), 797-803.

Mercuri, E., Messina, S., Battini, R., Berardinelli, A., Boffi, P., Bono, R., Bruno, C., Carboni, N., Cini, C., Colitto, F., D’Amico, A., Minetti, C., Mirabella, M., Mongini, T., Morandi, L., Dlamini, N., Orcesi, S., Pelliccioni, M., Pane, M., Pini, A., Swan, A.V., Villanova, M., Vita, G., Main, M., Muntoni, F., Bertini, E., 2006 Feb. Reliability of the Hammersmith functional motor scale for spinal muscular atrophy in a multicentric study. Neuromuscul. Disord. 16 (2), 93-98.

Mercuri, E., Bertini, E., Iannaccone, S.T., 2012. Childhood spinal muscular atrophy: controversies and challenges. Lancet Neurol. 11 (5), 443-452.

Munsat, T.L., Davies, K.E., 1992. Meeting report: International SMA consortium meeting, Bonn, 1992. Neuromuscul. Disord. 2, 423-428.
Perez, A., Mulot, R., Vardon, G., Barois, A., Gallego, J., 1996. Thoracoabdominal pattern of breathing in neuromuscular disorders. Chest 110 (2), 454-461.

Piepers, S., van den Berg, L.H., Brugman, F., et al., 2008. A natural history study of late onset spinal muscular atrophy types 3b and 4. J. Neurol. 255 (9), 1400-1404. Priori, R., Aliverti, A., Albuquerque, A.L., Quaranta, M., Albert, P., Calverley, P.M.A., 2013. The effect of posture on asynchronous chest wall movement in COPD. J. Appl. Physiol. 114 (8), 1066-1075.

Romei, M., Mauro, A.L., D’Angelo, M.G., Turconi, A.C., Bresolin, N., Pedotti, A., Aliverti, A., 2010. Effects of gender and posture on thoracoabdominal kinematics during quiet breathing in healthy adults. Respir. Physiol. Neurobiol. 172 (3), 184-191.

Romei, M. D'Angelo, M.G. LoMauro, A. Gandossini, S., Bonato, S., Brighina, E., Marchi, E., Comi, G.P., Turconi, A.C., Pedotti, A., Bresolin, N., Aliverti, A., 2012. Low abdominal contribution to breathing as daytime predictor of nocturnal desaturation in adolescents and young adults with Duchenne muscular dystrophy. Respir. Med. 106 (2), 276-283.

Russman, B.S., Iannacone, S.T., Buncher, C.R., Samaha, F.J., White, M., Perkins, B., Zimmerman, L., Smith, C., Burhans, K., Barker, L., 1992. Spinal muscular atrophy: new thoughts on the pathogenesis and classification schema. J. Child Neurol. 7 (4), 347-353.

Schroth, M.K., 2009. Special considerations in the respiratory management of spina muscular atrophy. Pediatrics 123 (4), S245-S249.

Talbot, K., 1999. Spinal muscular atrophy. J. Inherit. Metab. Dis. 22 (4), 545-554.

Wang, C.H., Finkel, R.S., Bertini, E.S., Schroth, M., Simonds, A., Wong, B., Aloysius, A. Morrison, L., Main, M., Crawford, T.O., Trela, A., Participants of the International Conference on SMA Standard of Care, 2007. Consensus statement for standard of care in spinal muscular atrophy. J. Child Neurol. 22 (8), 1027-1049.

Wee, C.D., Kong, L., Sumner, C.J., 2010. The genetics of spinal muscular atrophies. Curr. Opin. Neurol. 23 (5), 450-458. 